\title{
Quantitative characterization of acidic groups on acid-treated multi-walled carbon nanotubes using 1-aminopyrene as a fluorescent probe
}

\author{
Hiromasa Nishikiori, ${ }^{\text {a* }}$ Taku Tanigaki, ${ }^{a}$ Morinobu Endo, ${ }^{a}$ Tsuneo Fujii ${ }^{b}$
}

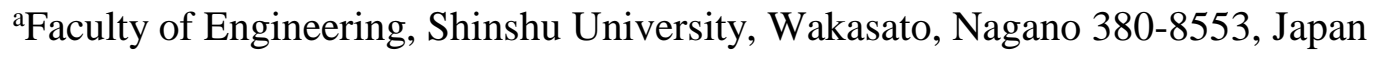

${ }^{b}$ Nagano Prefectural Institute of Technology, 813-8 Shimonogo, Ueda, Nagano 386-1211, Japan

\begin{abstract}
Acidic functional groups produced on the surface of acid-treated multi-walled carbon nanotubes (MWCNTs) were quantified by fluorescence measurements using 1-aminopyrene (1-AP) as an in-situ probe molecule. The 1-AP cation-like bands were observed on the $\mathrm{HNO}_{3} / \mathrm{H}_{2} \mathrm{SO}_{4}$ mixture-treated MWCNT surfaces because the 1-AP molecule was tightly immobilized by the hydrogen bonding interaction between its amino group and the Brönsted-acidic groups on the MWCNT surface. The fluorescence measurement allowed us to confirm the Langmuir-type adsorption of 1-AP on the functional groups of the MWCNTs, and estimate their amount of the functional groups and adsorption equilibrium constant. A longer acid treatment caused the chemical modification to generate higher amounts of the Brönsted-acidic functional groups and improve the adsorption ability on the MWCNT surface. About $2 \%$ of carbon in the MWCNTs was oxidized by the 24-h acid treatment. This value corresponded to $15-22 \%$ of carbon in the surface layer.
\end{abstract}

*Corresponding author. Tel: +81-26-269-5536 E-mail: nishiki@ shinshu-u.ac.jp (Hiromasa Nishikiori) 


\section{Introduction}

Advanced industry requires a nanotechnology to synthesize nanosized materials having a specific function. Carbon nanotubes (CNTs) are one of the most noteworthy materials for electronic, mechanical, and optical devices due to their unique structural and quantum characteristics [1-9]. Such carbon nanomaterials need to be functionalized on their surface in order to interact with other substances. For example, they should be well-dispersed in other raw materials, i.e., the incorporation of the carbon nanomaterials in plastics or ceramics provide practical materials with a certain shape and chemical and physical properties. Composites of matrices with dispersed CNTs were prepared, for example, by the sol-gel reaction of a system containing CNTs as the precursor chemical [10-12]. Surface functionalization of the carbon nanomaterials is an effective factor to allow them to easily interact and bond with various media for producing new functional materials utilizing their unique characteristics [13-15]. Therefore, CNTs can strongly interact with various matrices by pretreating their surface chemical structure with acids or other chemicals. The acid treatment of the carbon nanomaterials with nitric acid and sulfuric acid leads to the oxidation of their surface that forms oxidized groups such as $-\mathrm{COOH}$ and $-\mathrm{C}=\mathrm{O}$ in the graphene sheet [16-18]. Generally, the surface functional groups of the modified CNTs should be characterized by IR or Raman spectroscopy. However, it is difficult to obtain quantitative information about the chemical species existing in a monolayer or a few layers on the oxidized surface using these analyses. The quantification of the surface oxidized groups was performed by acid-base titration [19,20], XPS spectroscopy [20,21], and fluorescence measurement using fluorescent labeling [21-23]. The fluorescence measurement can more selectively and sensitively detect and quantify the oxygen containing functional groups of the nanoparticles than the other methods [22]. 
Carbon nanofibers (CNFs) [1,2,5], which are large-sized ones composed of multi-walled carbon nanotubes, are easier to be technologically and economically produced than single- or double-walled CNTs [3,4]. The CNFs are also important materials similar to the small-sized carbon nanotubes. We previously showed that observing the fluorescence spectra of 1-naphthol (1-NP) is a useful probe on a molecular level for studying the physicochemical properties of the surrounding environment around 1NP in various systems [24-29]. In situ fluorescence measurements using 1-NP as a physicochemical probe were performed in order to detect and quantify the adsorption sites on the surface of the CNFs dispersed in solvents and the sol-gel reaction systems of silicon alkoxide. This is a simple method not using a covalent-bonded probe molecule, but an adsorbed probe molecule. This measurements revealed that there are two adsorption forms; one is generated by the $\pi-\pi$ interaction between 1-NP and the graphene sheet and the other is the hydrogen-bonding interaction between 1-NP and the protonacceptable groups such as $-\mathrm{COOH}\left(-\mathrm{COO}^{-}\right)$and $-\mathrm{C}=\mathrm{O}[27-29]$ existing in a monolayer or a few layers on the surface. Our unique procedure to highly disperse the CNFs into solvents allowed these observations although the fluorescence of aromatic molecules adsorbed on carbon materials had never been observed due to its strong quenching. The fluorescence measurement allowed us to confirm the Langmuir-type adsorption of 1-NP on the graphene sheet and the proton-acceptable groups of the CNFs, and estimate the amounts of both sites and their adsorption equilibrium constants. The percentages of the oxidized carbon in the surface layer of the CNFs treated for 1 and $24 \mathrm{~h}$ were estimated to be $1.2 \%$ and $3.1 \%$, respectively [28].

1-Aminopyrene (1-AP) is a Brönsted base and is expected to interact with acidic groups on a solid surface [30,31]. In our previous study [32], oxygen-containing functional groups produced on the surface of the acid-treated CNFs have been characterized by UV-visible spectroscopy and the 
fluorescence measurements using 1-AP as a probe molecule in order to focus on such acidic groups such as $-\mathrm{COOH}[33,34]$. 1-AP has been found suitable as a fluorescence probe since its spectra drastically change with the acid-base equilibrium compared with those of aminonaphthalene or aminoanthracene. However, it was difficult to disperse a high amount of the CNFs in water which adsorb 1-AP enough to examine the quantitative adsorption properties of 1-AP. The acid-treated multi-walled carbon nanonubes (MWCNTs) with a smaller size are more easily dispersed in aqueous solvents. In this study, acidic functional groups on the surface of the acid-treated MWCNTs were quantified by fluorescence measurements using 1-AP in order to verify the validity of the in-situ fluorescent probing.

\section{Experimental}

1-AP (Wako, S grade) was recrystallized three times from water. Concentrated nitric acid, sulfuric acid, and sodium hydrogen carbonate (Wako, $\mathrm{S}$ grade) were used without further purification. The water was ion-exchanged and distilled. The MWCNTs (Iljin Nanotech, CM-95) have a diameter ca. 10-15 $\mathrm{nm}$ and a length of ca. $10-20 \mu \mathrm{m}$. To produce the functional groups on the surface of the MWCNTs, they were treated with the liquid acid [16-18,27,28]. As a typical procedure, the MWCNTs were refluxed in concentrated nitric acid at $393 \mathrm{~K}$ for $24 \mathrm{~h}$, rinsed with a large amount of water and dried at room temperature in vacuo. The untreated MWCNT sample was labeled N-CNT and that of the $\mathrm{HNO}_{3}$-treated was labeled A-CNT $[27,28]$. For a stronger treatment procedure, we applied a concentrated $\mathrm{H}_{2} \mathrm{SO}_{4} / \mathrm{HNO}_{3}$ mixture (3/1 in volume). First, the MWCNTs were irradiated with $28 \mathrm{kHz}$ ultrasonic waves in this mixture at $313 \mathrm{~K}$ for 1, 12, and $24 \mathrm{~h}$ (AIWA AU-10C). They were then refluxed in a mixture of concentrated sulfuric acid and $30 \%$ aqueous hydrogen peroxide (4/1 in volume) at $343 \mathrm{~K}$ for $24 \mathrm{~h}$. Finally, they were refluxed in concentrated nitric acid at $393 \mathrm{~K}$ for $24 \mathrm{~h}$. 
After finishing the process, they were rinsed with a large amount of water and dried at room temperature in vacuo. These MWCNT samples treated by the $\mathrm{H}_{2} \mathrm{SO}_{4}$ and $\mathrm{HNO}_{3}$ mixture for 1,12 , and $24 \mathrm{~h}$ were labeled AA-CNT-1h, $-12 \mathrm{~h}$, and $-24 \mathrm{~h}$, respectively. The surface structures of the untreated and three acid-treated MWCNT samples were hardly distinguished by their TEM images because only a surface layer was oxidized. These images agreed with previously reported results $[35,36]$.

The BET specific surface areas of the samples pretreated at $473 \mathrm{~K}$ for $12 \mathrm{~h}$ were measured by the volumetric gas adsorption method using nitrogen gas (BEL Japan BELSORP-mini). The samples were characterized by XPS using AlK $\alpha$ radiation (ULVAC PHI 5600). The AA-CNT-1h, $-12 \mathrm{~h}$, and -24h, $1.0 \mathrm{mg}$, were individually dispersed in water by ultrasonic irradiation for $24 \mathrm{~h}$. The suspension, $10 \mathrm{~cm}^{3}$, was titrated with the $1.0 \times 10^{-4} \mathrm{~mol} \mathrm{dm} \mathrm{daHCO}_{3}$ aqueous solution in order to estimate the number of the produced acidic functional groups on the MWCNT surface using a pH meter (TOA, HM-10P) [20].

The $\mathrm{N}$ - and AA-CNT-1h, $-12 \mathrm{~h}$, and $-24 \mathrm{~h}$ were individually dispersed in the 1-AP solutions containing water and ethanol $\left(8 / 2\right.$ in volume) at $1.0 \times 10^{-4} \mathrm{~mol} \mathrm{dm}^{-3}$ by ultrasonic irradiation for $1-18$ days. The resulting suspensions were centrifuged at $12,000 \mathrm{rpm}, 8,060 \mathrm{G}$ to remove any precipitates (IWAKI CFM-1000). The UV-visible absorption, fluorescence, and fluorescence excitation spectra of the resulting supernatant suspensions were then measured. The UV-visible absorption spectra were measured using a Shimadzu UV-3150 spectrophotometer, and the fluorescence and excitation spectra were measured using a Shimadzu RF-5300 spectrofluorophotometer. The excitation wavelength for the fluorescence spectra was $350 \mathrm{~nm}$, and the emission wavelength for the excitation spectra was 420 $\mathrm{nm}$. The concentrations of the AA-CNT-1h, $-12 \mathrm{~h}$, and $-24 \mathrm{~h}$ in the supernatant suspensions were estimated by measuring the weight of the residual MWCNTs after solvent evaporation at $473 \mathrm{~K}$ for 12 h. 


\section{Results and discussion}

\subsection{Changes in $U V$-visible absorption spectra of MWCNT suspensions}

The adsorption of 1-AP on the MWCNT samples and the dispersion of the MWCNTs into the liquid phase were examined by measuring the UV-visible absorption spectra of the MWCNT suspensions. The absorption spectra of the N-CNT and AA-CNT-1h, -12h, and -24h suspensions containing 1-AP were observed just after their preparation, and after the ultrasonic irradiation for $2-18$ days. In the NCNT suspension, the spectrum was only slightly changed with time. Apparently, 1-AP was not absorbed on the N-CNT surface. In a previous study, the 1-AP molecules were only slightly adsorbed on the N-CNF surface similar to 1-NP [27,28,32]. The N-CNF adsorbed pyrene better than 1-AP [37]. This result indicates that the polar amino group prevents the $\pi-\pi$ interaction between the pyrene ring and graphene sheet although pyrene must be adsorbed on the carbon nanotubes by this interaction $[38,39]$. In the AA-CNT suspension, the absorbance gradually decreases with time. It was confirmed that 1-AP interacts with the acid-treated MWCNTs similar to the acid-treated CNFs. The change in the absorption spectrum of the AA-CNT-24h suspension is shown in Figure 1 as a typical example.

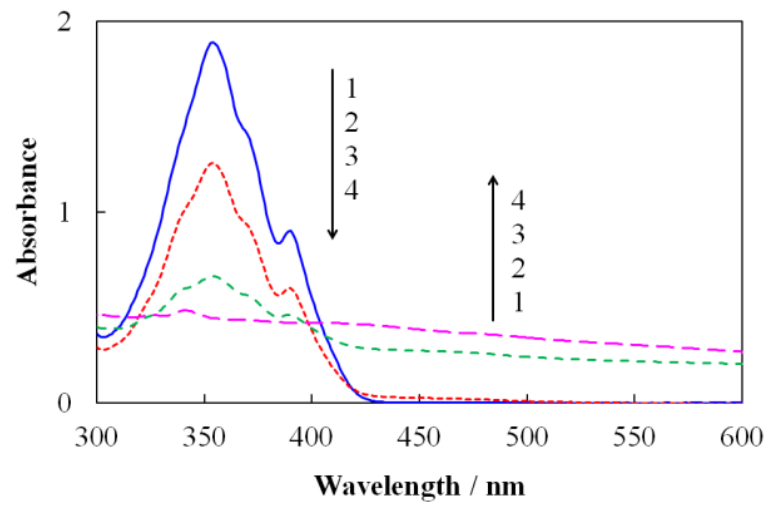

Figure 1 Absorption spectra of AA-CNT-24h suspension containing 1-AP observed (1) just after the preparation and after ultrasonic irradiation of (2) 2 days, (3) 6 days, and (4) 8 days. 
Figure 2 shows the changes in the absorbance of each MWCNT suspension at 350 and $500 \mathrm{~nm}$ plotted versus the ultrasonic irradiation time. The absorbance at $350 \mathrm{~nm}$ indicates the amount of 1-AP existing in the liquid phase. 1-AP was hardly adsorbed on the N-CNT, but was adsorbed on the AACNTs. The AA-CNTs acid-treated for a longer time can adsorb 1-AP faster. The absorbance at 500 $\mathrm{nm}$ is regarded to correspond to the degree of the MWCNT dispersion according to a good correlation between the concentration and the absorbance of the carbon nanotubes in a solvent [40]. The absorbance increased with the ultrasonic irradiation time and reached saturation. These results indicated that the order of the degree of the MWCNT dispersion was that of the acid treatment duration and it is closely correlated to the amount of adsorbed 1-AP. Not only the surface modification by the acid treatment, but also the adsorption of aromatic molecules should play an important role in the MWCNT dispersion [28].

(a)

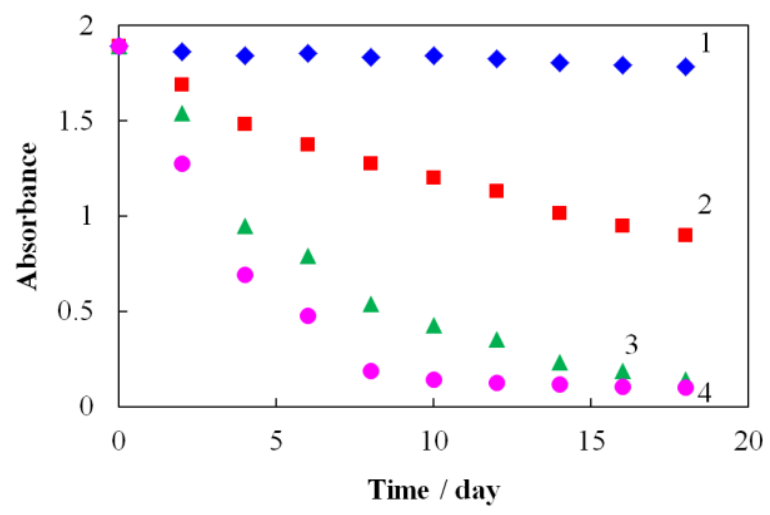

(b) 


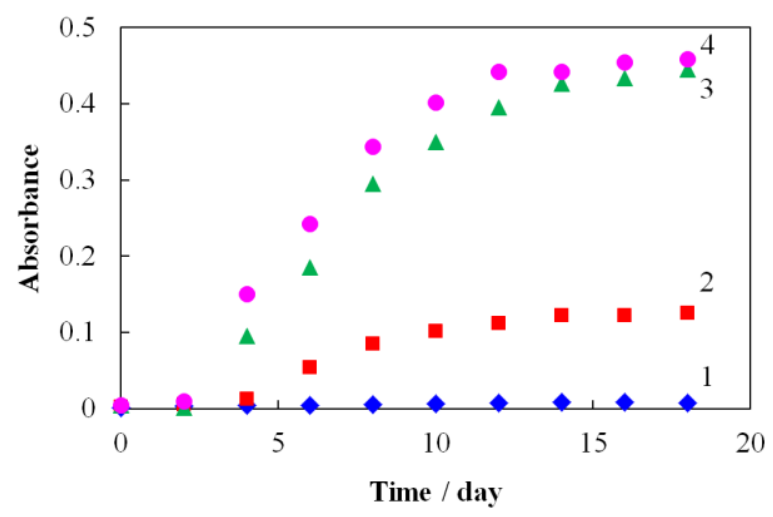

Figure 2 Changes in absorbance vs. ultrasonic irradiation time monitored at (a) 350 and (b) $500 \mathrm{~nm}$ in (1) N-CNT, (2) AA-CNT-1h, (3) AA-CNT-12h, and (4) AA-CNT-24h suspensions.

\subsection{Changes in fluorescence spectra of MWCNT suspensions}

1-AP exhibits a protonation equilibrium; the original species is called AP, and the protonated species is called $\mathrm{APH}^{+}$. The protonation equilibrium constant of the ground state, $\mathrm{pK}_{\mathrm{a}}$, and the excited state, $\mathrm{pK}_{\mathrm{a}}{ }^{*}$, are 2.8 and -1.2 , respectively [41]. Therefore, in somewhat low-pH solutions, 1-AP existing as $\mathrm{APH}^{+}$in the ground state, is deprotonated to form AP in the excited states, and emits a fluorescence.

Figures 3 and 4 show the fluorescence spectra of 1-AP in the N-CNT and AA-CNT-1h suspensions, respectively, observed just after the preparation and after the ultrasonic irradiation. The fluorescence spectral band at around $440 \mathrm{~nm}$ is assigned to AP present in liquid phase of the suspension [30,31]. The spectrum of the N-CNT suspension only slightly changed even after the ultrasonic irradiation of 18 days. The fluorescence spectral bands at around 360-400 nm observed in the AA-CNT-1h suspension are similar to those of $\mathrm{APH}^{+}$in the acidic solution [30,31], and their relative intensities increased with the ultrasonic irradiation time. These results indicated that 1-AP is absorbed on the MWCNT surface to form $\mathrm{APH}^{+}$-like species similar to that on CNF surface. 


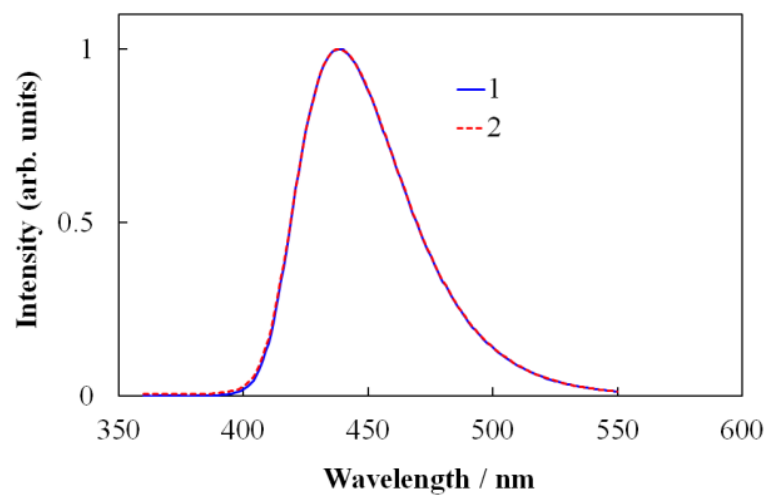

Figure 3 Fluorescence spectra of 1-AP in N-CNT suspension observed (1) just after the preparation and after ultrasonic irradiation of (2) 18 days.

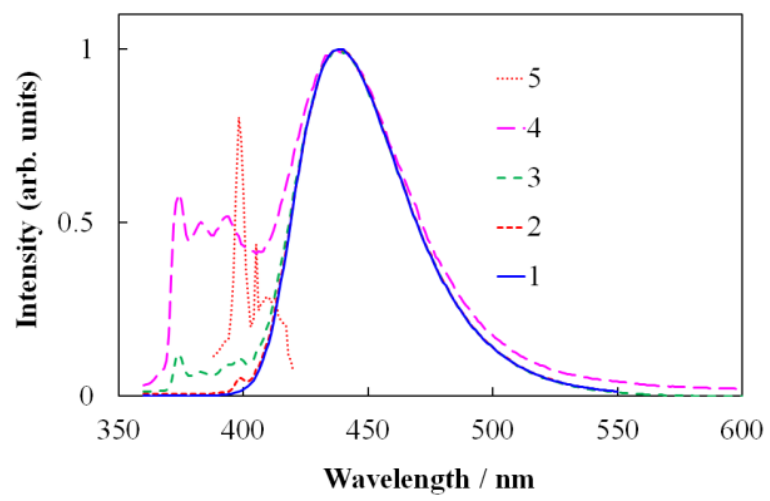

Figure 4 Fluorescence spectra of 1-AP in AA-CNT-1h suspension observed (1) just after the preparation and after the ultrasonic irradiation of (2) 1, (3) 4, and (4) 11 days with (5) the differential spectrum between the spectra (1) and (2).

A peak was observed at $398 \mathrm{~nm}$ after 1 day (spectrum 2). The differential spectrum (5) from that observed just after preparation (1) exhibited the peaks at 398, 405, and $415 \mathrm{~nm}$. This is similar to the spectrum of 1-AP in cyclohexane having peaks at 399, 405, and $420 \mathrm{~nm}$ [30]. In a previous study, the ${ }^{1} \mathrm{Lb}_{b}$ fluorescence of 1-NP, which is observed in nonpolar environments, was seen on the CNFs due to 
the $\pi-\pi$ interaction between 1-NP and the graphene sheet [27-29]. A large amount of 1-AP molecules must also be adsorbed on the graphene sheet of the acid-treated CNFs because they are better dispersed in the liquid phase than the N-CNF $[35,36]$. Such a fluorescence was temporally observed in the present system containing 1-AP, and then hidden by the strong $\mathrm{APH}^{+}$-like band.

It was previously reported that the ion-pair fluorescence of 1-NP was generated by the relatively strong hydrogen-bonding interaction between $1-\mathrm{NP}$ and the oxidized groups, such as $-\mathrm{COOH},-\mathrm{C}=\mathrm{O}$, and $-\mathrm{OH}[27,28]$. A Brönsted base, 1-AP, is expected to interact with the acidic oxygen-containing groups. Therefore, 1-AP selectively detected the acidic groups such as - $\mathrm{COOH}$ [32].

\subsection{Quantitative characteristics of 1-AP adsorbed on acid-treated MWCNTs}

The physicochemical properties for the MWCNT samples were determined by conventional methods. Table 1 shows the BET specific surface area, ratio of $\mathrm{C} / \mathrm{O}$ atoms, and concentration of the adsorption sites for each sample estimated by the BET method, XPS analysis, and $\mathrm{NaHCO}_{3}$ titration, respectively. The specific surface area of the MWCNTs was not significantly changed by the acid treatment. The surface structures of the samples were hardly distinguished by their TEM images because only a surface layer was oxidized as previously reported [35,36]. Based on the results of the XPS analysis and $\mathrm{NaHCO}_{3}$ titration, the relative amount of oxygen atom and concentration of the adsorption sites reasonably increased with an increase in the acid-treatment time. However, in the XPS analysis, it was difficult to distinguish some oxygen-containing groups because the amount of oxygen atom was very low. These results indicated that Brönsted-acidic functional groups were produced on the acid-treated MWCNT surface and their number increased with the treatment time.

Table 1 Physicochemical properties for the MWCNT samples determined by conventional methods 


\begin{tabular}{cccc}
\hline & $\begin{array}{c}\text { Specific surface } \\
\text { area } / \mathrm{m}^{2} \mathrm{~g}^{-1}\end{array}$ & Ratio of C/O atoms & $\begin{array}{c}\text { Concentration of adsorption } \\
\text { sites } / \mathrm{mol} \mathrm{g}^{-1}\end{array}$ \\
\hline N-CNT & 200 & $98.3 / 1.7$ & - \\
AA-CNT-1h & 210 & $97.3 / 2.7$ & $4.0 \times 10^{-5}$ \\
AA-CNT-12h & 220 & $96.1 / 3.9$ & $6.0 \times 10^{-5}$ \\
AA-CNT-24h & 240 & $94.5 / 5.5$ & $1.9 \times 10^{-4}$ \\
\hline
\end{tabular}

Figure 5 shows the fluorescence spectra of 1-AP observed in aqueous suspensions including the AACNTs prepared at different initial 1-AP concentrations. The fluorescence intensity of the $\mathrm{APH}^{+}$-like species relative to that of the AP decreases with an increase in the 1-AP concentration, i.e., the ratio of 1-AP in the liquid phase becomes higher, indicating that the adsorption approaches an equilibrium state. The Langmuir-type adsorption of 1-AP on the functional groups of the MWCNTs was assumed in this study. The total concentration of $1-\mathrm{AP}, C$, is written as

$$
C=[\mathrm{AP}]+\left[\mathrm{APH}^{+}\right]
$$

where $\mathrm{AP}$ and $\mathrm{APH}^{+}$are 1-AP existing in the liquid phase and on the MWCNT surface, respectively. The ratio of the fluorescence intensity of $\mathrm{AP}, I_{\mathrm{AP}}$, to that of $\mathrm{APH}^{+}, I_{\mathrm{APH}}$, is defined as $R$. The desorption in the excited states can be ignored because the diffusion rate of molecules is much lower than the fluorescence lifetime.

$$
R=\frac{I_{\mathrm{AP}}}{I_{\mathrm{APH}+}}=A \frac{[\mathrm{AP}]}{\left[\mathrm{APH}^{+}\right]}
$$

where $A$ indicates the ratio of the spectroscopic constant of AP to that of $\mathrm{APH}^{+}$defined by the molar extinction coefficient, $\varepsilon$, and fluorescence quantum yield, $\Phi$.

$$
A=\frac{\varepsilon_{\mathrm{AP}}}{\varepsilon_{\mathrm{APH}+}} \times \frac{\Phi_{\mathrm{AP}}}{\Phi_{\mathrm{APH}+}}
$$


These equations can be applied to the Langmuir isotherm as follows.

$$
\theta=\frac{\left[\mathrm{APH}^{+}\right]}{[\mathrm{F}]}=\frac{K[\mathrm{AP}]}{1+K[\mathrm{AP}]}
$$

where $\theta$ is the fractional surface coverage and $\mathrm{F}$ is the adsorption site due to the $\pi-\pi$ interaction on the AA-CNT surface, and $K$ is the adsorption equilibrium constant on the $\mathrm{F}$ site. The value $[\mathrm{F}]$ is the molar amount of the F sites existing on $1 \mathrm{~g}$ of the MWCNTs. In order to determine the quantitative adsorption properties of the AA-CNTs, the MWCNT concentrations in the suspensions should be considered to depend on the 1-AP concentration. Figure 6 shows the MWCNT concentrations observed as a function of the initial concentration of 1-AP in the AA-CNT suspensions. The amount of the 1-AP adsorbed on the AA-CNTs was corrected to that per $1 \mathrm{~g}$ of the AA-CNTs.

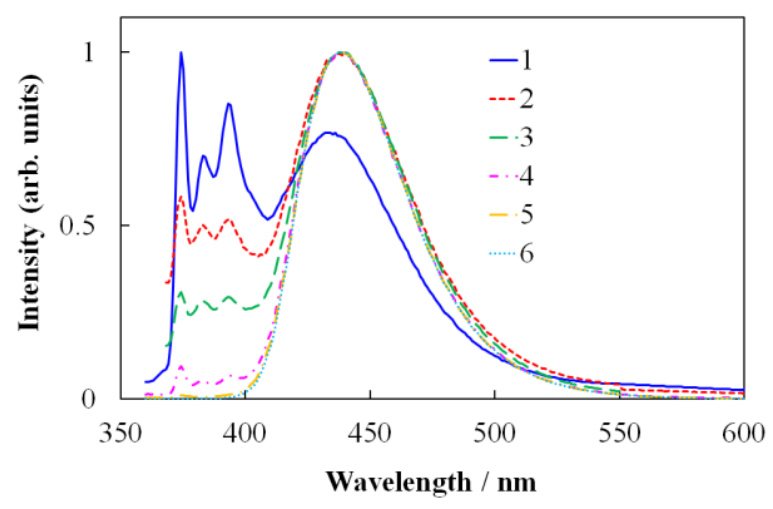

Figure 5 Fluorescence spectra of 1-AP in AA-CNT-24h suspensions, in which the initial concentrations of 1 -AP are (1) $8.0 \times 10^{-6}$, (2) $5.0 \times 10^{-5}$, (3) $8.0 \times 10^{-5}$, (4) $1.0 \times 10^{-4}$, (5) $2.0 \times 10^{-4}$, and (6) $5.0 \times 10^{-4} \mathrm{M}$, observed after ultrasonic irradiation of 18 days. The excitation wavelength is $350 \mathrm{~nm}$. 


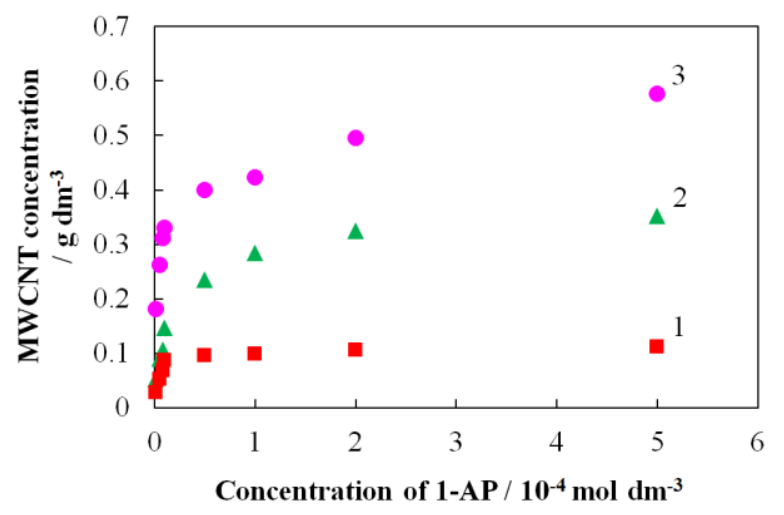

Figure 6 Dependence of the MWCNT concentrations in (1) AA-CNT-1h, (2) AA-CNT-12h, and (3) AA-CNT-24h suspensions on the initial concentration of 1-AP.

The Langmuir isotherm of 1-AP on the surface of the AA-CNTs was obtained by the curve fitting calculation using the known variables, $C$ and $R$, and the parameters, $A, K$, and $[\mathrm{F}]$. The experimental and simulated relationships between $\theta$ and [AP] are plotted in Figure 7. The experimental values are well-fitted to the Langmuir isotherm. The resulting constants of $A, K$, and $[\mathrm{F}]$ are summarized in Table 2. It was confirmed that a longer acid treatment caused the chemical modification to generate higher amounts of the Brönsted-acidic functional groups and improve the adsorption ability on the MWCNT surface. The constants should be the average values for the adsorption sites of various Brönsted-acidic functional groups. The concentrations of the adsorption sites for the samples were similar to those determined by the $\mathrm{NaHCO}_{3}$ titration. About $2 \%$ of carbon in the MWCNTs was oxidized by the 24-h acid treatment based on the molar amount of $1 \mathrm{~g}$ carbon, $8.3 \times 10^{-2} \mathrm{~mol}$. The XPS analysis indicated that the oxygen percentage for AA-CNT-24h was 5.5\%. The oxidized carbon percentage for AACNT-24h can be estimated to be about $2 \%$ if all the oxidized carbons were assumed to form carboxyl groups. The present fluorescence measurement quantified the functional groups existing in the surface layer. The percentage of the surface carbons to all the carbons in the present MWCNT is $9-13 \%$. 
Parts of the AA-CNT-24h surface layer, 15-22\%, were presumed to be oxidized on which the functional groups were produced.

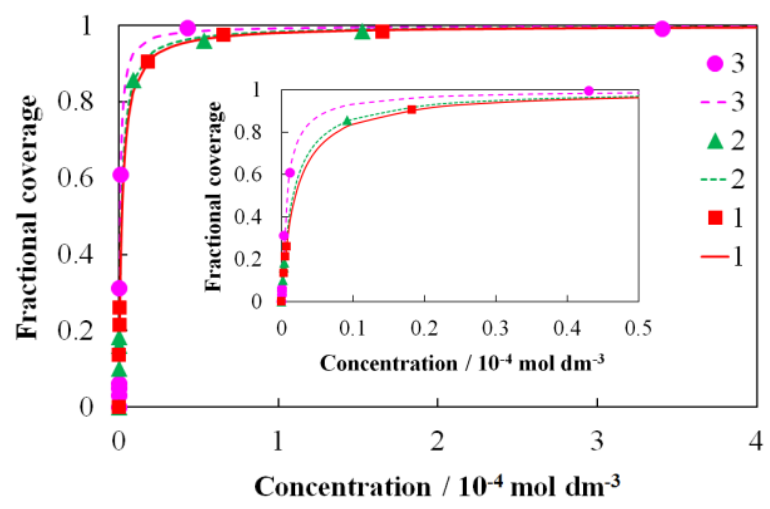

Figure 7 Langmuir isotherm for 1-AP adsorption on (1) AA-CNT-1h, (2) AA-CNT-12h, and (3) AACNT-24h suspensions.

Table 2 Constants for the acid-treated MWCNT samples estimated by fluorescence measurements using 1-AP

\begin{tabular}{cccc}
\hline & $\begin{array}{c}\text { Spectroscopic } \\
\text { constant }\end{array}$ & $\begin{array}{c}\text { Adsorption equilibrium } \\
\text { constant } / \mathrm{mol}^{-1} \mathrm{dm}^{3}\end{array}$ & $\begin{array}{c}\text { Concentration of adsorption } \\
\text { sites } / \mathrm{mol} \mathrm{g}^{-1}\end{array}$ \\
\hline AA-CNT-1h & 48 & $5.1 \times 10^{5}$ & $3.5 \times 10^{-4}$ \\
AA-CNT-12h & 66 & $6.2 \times 10^{5}$ & $4.8 \times 10^{-4}$ \\
AA-CNT-24h & 86 & $1.4 \times 10^{6}$ & $1.6 \times 10^{-3}$ \\
\hline
\end{tabular}

\section{Conclusions}

Brönsted-acidic functional groups produced on the acid-treated MWCNT surface were quantified using 1-AP as a fluorescent probe. 1-AP was adsorbed on the MWCNT surface by the hydrogen- 
bonding interaction between the amino group of 1-AP and the acidic functional group, such as $-\mathrm{COOH}$ on the MWCNT surface, to form $\mathrm{APH}^{+}$-like species. The Langmuir-type adsorption of 1-AP on the functional groups of the MWCNTs was confirmed and their amounts and adsorption equilibrium constants were determined. A longer acid treatment caused the chemical modification to generate higher amounts of the acidic functional groups and improve the adsorption ability on the MWCNT surface. The MWCNT sample having a higher amount of functional groups was better dispersed in the 1-AP solution, and adsorbed a higher amount of 1-AP. About $2 \%$ of carbon in the MWCNTs was oxidized by the $24-\mathrm{h}$ acid treatment. This value corresponded to $15-22 \%$ of carbon in the surface layer. The present fluorescence measurement quantified the functional groups existing in the surface layer. The number of the oxidized carbons was consistent with that determined by XPS analysis and $\mathrm{NaHCO}_{3}$ titration.

\section{References}

[1] Oberlin A, Endo M, Koyama T. Filamentous growth of carbon through benzene decomposition. J Cryst Growth 1976; 32(3): 335-349.

[2] Endo M. Grow carbon fibers in the vapor phase. Chemtech. 1988; 18(9): 568-576.

[3] Iijima S. Helical microtubules of graphitic carbon. Nature 1991; 354(6348): 56-58.

[4] Iijima S, Ichihashi T. Single-shell carbon nanotubes of 1-nm diameter. Nature 1993; 363(6430): 603-605.

[5] Endo M, Kim YA, Hayashi T, Nishimura K, Matsushita T, Dresselhaus MS. Vapor-grown carbon fibers (VGCFs): Basic properties and their battery applications. Carbon 2001; 39(9): $1287-1297$. 
[6] Endo M, Kim YA, Fukai T, Hayashi T, Oshida K, Terrones M, et al. Structural characterization of cup-stacked-type nanofibers with an entirely hollow core. Appl Phys Lett 2002; 80(7): 12671269.

[7] Yang X, Guillorn MA, Austin D, Melechko AV, Cui H, Meyer III HM, et al. Fabrication and characterization of carbon nanofiber-based vertically integrated schottky barrier junction diodes. Nano Lett 2003; 3(12): 1751-1755.

[8] Wang F, Arai S, Endo M. Preparation of nickel-carbon nanofiber composites by a pulse-reverse electrodeposition process. Electrochem Commun 2005; 7(7): 674-678.

[9] Tan TT, Sim HS, Lau SP, Yang HY, Tanemura M, Tanaka J. X-ray generation using carbonnanofiber-based flexible field emitters. Appl Phys Lett 2006; 88(10): 103105-1-103105-3.

[10] Wang S, Ji L, Wu B, Gong Q, Zhu Y, Liang J. Influence of surface treatment on preparing nanosized $\mathrm{TiO}_{2}$ supported on carbon nanotubes. Appl Surf Sci 2008; 255(5): 3263-3266.

[11] Jung I, Gurav JL, Bangi UKH, Baek S, Park HH. Silica xerogel films hybridized with carbon nanotubes by single step sol-gel processing. J Non-Crystalline Solids 2012; 358(3): 550-556.

[12] Omidvar H, Mirzaei FK, Rahimi MH, Sadeghian Z. A method for coating carbon nanotubes with titanium. New Carbon Mater 2012; 27(6): 401-408.

[13] Zhu J, Kim JD, Peng H, Margrave JL, Khabashesku VN, Barrera EV. Improving the dispersion and integration of single-walled carbon nanotubes in epoxy composites through functionalization. Nano Lett 2003; 3(8): 1107-1113.

[14] Gao J, Itkis ME, Yu A, Bekyarova E, Zhao B, Haddon RC. Continuous spinning of a singlewalled carbon nanotube-nylon composite fiber. J Am Chem Soc 2005; 127(11) 3847-3854. 
[15] Singh R, Pantarotto D, McCarthy D, Chaloin O, Hoebeke J, Partidos CD, et al. Binding and condensation of plasmid DNA onto functionalized carbon nanotubes: Toward the construction of nanotube-based gene delivery vectors. J Am Chem Soc 2005; 127(12): 4388-4396.

[16] Liu J, Rinzer AG, Dai H, Hafner JH, Bradley RK, Boul PJ, et al. Fullerene pipes. Science 1998; 280(5367): 1253-1256.

[17] Hamon MA, Hui H, Bhowmik P, Niyogi S, Zhao B, Itkis ME, et al. End-group and defect analysis of soluble single-walled carbon nanotubes. Chem Phys Lett 2001; 347(1-3): 8-12.

[18] Hamon MA, Hui H, Bhowmik P, Itkis HME, Haddon RC. Ester-functionalized soluble singlewalled carbon nanotubes. Appl Phys A 2002; 74(3): 333-338.

[19] Hanelt S, Orts-Gil G, Friedrich JF, Meyer-Plath A. Differentiation and quantification of surface acidities on MWCNTs by indirect potentiometric titration. Carbon 2011; 49(9): 2978-2988.

[20] Gong H, Kim ST, Lee JD, Yim S. Simple quantification of surface carboxylic acids on chemically oxidized multi-walled carbon nanotubes. Appl Surf Sci 2013; 266: 219224.

[21] Pellenbarg T, Dementev N, Jean-Gilles R, Bessel C, Borguet E, Dollahon N, et al. Detecting and quantifying oxygen functional groups on graphite nanofibers by fluorescence labeling of surface species. Carbon 2010; 48(15): 4256-4267

[22] Dementev N, Feng X, Borguet E. Fluorescence labeling and quantification of oxygencontaining functionalities on the surface of single-walled carbon nanotubes. Langmuir 2009; 25(13): 7573-7577.

[23] Dementev N, Ronca R, Borguet E. Oxygen-containing functionalities on the surface of multiwalled carbon nanotubes quantitatively determined by fluorescent labeling. Appl Surf Sci 2012; 258: $10185-10190$. 
[24] Suzuki S, Fujii T, Imai A, Akahori H. The fluorescent level inversion of dual fluorescences and the motional relaxation of excited state molecules in solutions. J Phys Chem 1977; 81(16): $1592-1598$.

[25] Fujii T, Mabuchi T, Kitamura H, Kawauchi O, Negishi N, Anpo M. Fluorescence spectra of 1naphthol during the sol-gel process of a mixed aluminum-silicon alkoxide ( $\mathrm{Si}: \mathrm{Al}=94: 6$ ). Bull Chem Soc Jpn 1992; 65(3): 720-727.

[26] Fujii T, Sugawara Y, Kodaira K, Mabuchi T, Anpo M. Characteristic changes in the fluorescence spectra of 1-naphthol at the gelation point during the sol-gel-xerogel transitions. Res Chem Intermed 1995; 21(6): 643-652.

[27] Nishikiori H, Tanaka N, Kubota S, Endo M, Fujii T. In situ characterization of surface physicochemical properties of carbon nanofibers using 1-naphthol as a fluorescent probe. Chem Phys Lett 2004; 390(4-6): 389-393.

[28] Kubota S, Nishikiori H, Tanaka N, Endo M, Fujii T. Quantitative characterization of surface adsorption sites of carbon nanofibers by in-situ fluorescence measurement using 1-naphthol. Chem Phys Lett 2005; 412(1-3): 223-227.

[29] Kubota S, Nishikiori H, Tanaka N, Endo M, Fujii T. Dispersion of acid-treated carbon nanofibers into gel matrices prepared by the sol-gel method. J Phys Chem B 2005; 109(49): 23170-23174.

[30] Hite P, Krasnansky R, Thomas JK. Spectroscopic investigations of surfaces by using aminopyrene. J Phys Chem 1986; 90(22): 5795-5799.

[31] Miller E, Wysocki S, Jóźwik D. Fluorescence studies of the sol-gel transition using aminopyrene. J Photochem Photobiol A 2005; 169(3): 221-228. 
[32] Nishikiori H, Tanaka N, Tanigaki T, Endo M, Fujii T. In situ probing of acidic groups on acidtreated carbon nanofibers using 1-aminopyrene. J Photochem Photobiol A: Chem 2008; 193(23): $161-165$.

[33] Chen J, Rao AM, Lyuksyutov S, Itkis ME, Hamon MA, Hu H, et al. Dissolution of full-length single-walled carbon nanotubes. J Phys Chem B 2001; 105(13): 2525-2528.

[34] Kahn MG, Banerjee S, Wong SS. Solubilization of oxidized single-walled carbon nanotubes in organic and aqueous solvents through organic derivatization. Nano Lett 2002; 2(11): 12151218.

[35] Toebes ML, van Heeswijk JMP, Bitter JH, van Dillen AJ, de Jong KP. The influence of oxidation on the texture and the number of oxygen-containing surface groups of carbon nanofibers. Carbon 2004; 42(2): 307-315.

[36] Lakshminarayanan PV, Toghiani H, Pittman Jr. CU. Nitric acid oxidation of vapor grown carbon nanofibers. Carbon 2004; 42(12-13): 2433-2442.

[37] Tanigaki T, Nishikiori H, Kubota S, Tanaka N, Endo M, Fujii T. Fluorescence observation of pyrene adsorbed on carbon nanofibers. Chem Phys Lett 2007; 448(4-6): 218-222.

[38] Long RQ, Yang RT. Carbon nanotubes as superior sorbent for dioxin removal. J Am Chem Soc 2001; 123(9): 2058-2059.

[39] Chen RJ, Zhang Y, Wang D, Dai H. Noncovalent sidewall functionalization of single-walled carbon nanotubes for protein immobilization. J Am Chem Soc 2001; 123(16): 3838-3839.

[40] Bahr JL, Mickelson ET, Bronikowski MJ, Smalley RE, Tour JM. Dissolution of small diameter single-wall carbon nanotubes in organic solvents? Chem Commun 2001; 2001(2): 193-194. 
[41] Shizuka H, Tsutsumi K, Takeuchi H, Tanaka I. Direct measurement of proton dissociation in the excited state of protonated 1-aminopyrene with picosecond pulses. Chem Phys Lett 1979; 62(2): 408-411. 